\title{
TECNOLOGIA EDUCACIONAL EM SAÚDE: CONTRIBUIÇÕES PARA A ENFERMAGEM PEDIÁTRICA E NEONATALa
}

\author{
Educational technology in health: contributions for pediatric and neonatal nursing
}

Tecnología educativa en salud: contribuciones para la enfermería pediátrica y neonatal

Luciana Mara Monti Fonseca'

Marta Angélica lossi Silva ${ }^{4}$
Adriana Moraes Leite ${ }^{2}$

Regina Aparecida Garcia de Lima ${ }^{5}$
Débora Falleiros de Mello ${ }^{3}$

Carmen Gracinda Silvan Scochi ${ }^{6}$

\section{RESUMO}

Vislumbra-se o uso da tecnologia educacional, como recurso facilitador para o ensino e prática de enfermagem pediátrica e neonatal. Na tentativa de oferecer uma aprendizagem ao estudante, à equipe de enfermagem e à criança e sua família mais motivadora, tem-se feito uso de recursos tecnológicos por meio dos materiais educativos. Neste relato, descreve-se a experiência de um grupo de estudos e pesquisa na produção de materiais voltados para a formação e educação permanente na área de enfermagem pediátrica e neonatal como também na educação em saúde de crianças e seus familiares sobre diferentes temas que permeiam essa assistência. Considera-se que os materiais educacionais criados favorecem que estudantes, profissionais de saúde e clientes vivenciem o processo ensino-aprendizagem de forma estimulante, facilitando o esclarecimento de dúvidas.

Palavras-chave: Educação em Enfermagem. Educação em Saúde. Tecnologia Educacional. Enfermagem Pediátrica. Enfermagem Neonatal.

\begin{abstract}
The use of educational technology, as a facilitating resource for pediatric and neonatal nursing teaching and practice, is pointed out. Technological resources in educational materials have been used in a progressively motivating and interactive pedagogical relationship, aiming to offer a more motivating learning for students, nursing teams, children and their families. This report describes the experience of a research and study group in the development of materials targeting permanent training and education in the area of pediatric and neonatal nursing. Health education for children and their relatives on different themes that permeates this care was also targeted. The developed educational materials permit students, health professionals and clients to experience the teaching and learning process in a stimulating way, facilitating the clarification of doubts and transforming learning through attractive, innovative and illustrative situations.
\end{abstract}

Keywords: Education, Nursing. Health Education. Educational Technology. Pediatric Nursing. Neonatal Nursing.

\section{Resumen}

Se señala el uso de la tecnología educativa como recurso facilitador para la enseñanza y la práctica de enfermería pediátrica y neonatal. En el intento de ofrecer un aprendizaje más motivador al estudiante, al equipo de enfermería, y a los niños y sus familias, los recursos tecnológicos son usados por medio de materiales educativos en una relación pedagógica progresivamente motivadora e interactiva. Este informe describe la experiencia de un grupo de estudios e investigación en la producción de materiales dirigidos a la formación y educación permanente en el área de enfermería pediátrica y neonatal, así como la educación en salud de niños y sus familiares acerca de diferentes temas que permean esa atención. Los materiales educacionales criados permiten que estudiantes, profesionales de salud y clientes experimenten el proceso enseñanza-aprendizaje de forma estimulante, facilitando la aclaración de dudas y trasformando el aprendizaje a través de situaciones atractivas, innovadoras e ilustrativas.

Palabras clave: Educación en Enfermería. Educación en Salud. Tecnología Educacional. Enfermería Pediátrica. Enfermería Neonatal.

\footnotetext{
${ }^{1}$ Enfermeira. Professora Doutora do Departamento de Enfermagem Materno-Infantil e Saúde Pública da Escola de Enfermagem de Ribeirão Preto da Universidade de São Paulo. Ribeirão Preto-SP. Brasil. E-mail: lumonti@eerp.usp.br²Enfermeira. Professora Doutora do Departamento de Enfermagem Materno-Infantil e Saúde Pública da Escola de Enfermagem de Ribeirão Preto da Universidade de São Paulo. Ribeirão Preto - SP. Brasil. E-mail: drileite@eerp.usp.brEnfermeira. Professora Associada do Departamento de Enfermagem Materno-Infantil e Saúde Pública da Escola de Enfermagem de Ribeirão Preto da Universidade de São Paulo. Ribeirão Preto-SP. Brasil. E-mail: defmello@eerp.usp.brEnfermeira. Professora Doutora do Departamento de Enfermagem Materno-Infantil e Saúde Pública da Escola de Enfermagem de Ribeirão Preto da Universidade de São Paulo. Ribeirão Preto - SP. Brasil. E-mail: maiossi@eerp.usp.br, ${ }^{5}$ Enfermeira. Professora Titular do Departamento de Enfermagem Materno-Infantil e Saúde Pública da Escola de Enfermagem de Ribeirão Preto da Universidade de São Paulo. Ribeirão PretoSP. Brasil. E-mail: limare@eerp.usp.br, EEnfermeira. Professora Titular do Departamento de Enfermagem Materno-Infantil e Saúde Pública da Escola de Enfermagem de Ribeirão Preto da Universidade de São Paulo. Ribeirão Preto - SP. Brasil. E-mail: cscochi@eerp.usp.br
} 


\section{INTRODUÇÃO}

A assistência de enfermagem à criança e ao neonato é algo complexo. Além da execução adequada das técnicas de enfermagem, exige do enfermeiro uma gama de competências que vão desde o conhecimento sobre as patologias mais frequentes em cada período do desenvolvimento até as necessidades sociais e emocionais da criança e sua família.

Na formação de enfermeiros para o cuidado à criança e ao adolescente, apesar do avanço tecnológico, o ensino no Brasil ainda ocorre, em sua maioria, de forma tradicional, ministrando-se aulas expositivas e sem a participação efetiva dos estudantes. Por outro lado, já se observam mudanças em face às novas diretrizes curriculares do ensino superior em que é recomendado o uso adequado de tecnologias da informação, da comunicação e de outras que possam colaborar no cuidado em enfermagem. Neste contexto, consideramos que 0 desenvolvimento de materiais educacionais pode contribuir com um ensino mais participativo disponibilizando para os estudantes conteúdos que poderão ser utilizados de acordo com suas necessidades e ritmos de aprendizagem. Esse avanço tecnológico também pode apoiar o cotidiano do enfermeiro, disponibilizando informações e potencializando a aquisição de conhecimentos, na educação permanente deste profissional.

Nossa experiência no desenvolvimento, validação e utilização de materiais educacionais voltados para a formação, educação permanente de enfermeiros e para a educação em saúde junto à clientela, têm evidenciado que a crescente evolução de tecnologias a partir das demandas da sociedade apresenta novas possibilidades de uso destes materiais no cotidiano do trabalho em instituições de ensino e saúde.

$\mathrm{Na}$ enfermagem brasileira, o desenvolvimento de programas de ensino mediado por tecnologias constitui, na atualidade, uma tendência crescente e está vinculado às universidades com projetos de pesquisas dirigidos, predominantemente, à formação dos graduandos e educação em saúde da clientela.

A enfermagem tem se envolvido com a produção e busca de artifícios tecnológicos para auxiliar no seu cotidiano profissional, permeando suas atividades assistenciais, administrativas e educacionais. ${ }^{2}$ Assim, temos observado um aumento na produção de tecnologias pela enfermagem, ainda que pouco divulgada.

Sabemos que as dificuldades e escassez de recursos físicos, humanos, estruturais e materiais em grande parcela dos serviços de saúde tornam a prática educativa monótona, desestimulante e repetitiva, para o profissional e para a clientela. Acreditamos que os materiais de ensino dinamizam as atividades de Educação em Saúde. Desta forma, vislumbramos o uso da tecnologia educacional como recurso facilitador para o ensino e prática de enfermagem pediátrica e neonatal.

Pensamos que, ao apresentar para estudantes, profissional de enfermagem e clientela novas formas de aprender e ensinar, por meio do relato da produção dessas tecnologias educacionais criativas e inovadoras, instigando-os a criar e usar os materiais educacionais no seu cotidiano, esperamos contribuir com a formação e educação permanente de enfermeiros e a educação em saúde de crianças e seus familiares sobre diferentes temas que permeiam a assistência de enfermagem pediátrica e neonatal.

\section{OBJ ETIVO}

Descrever as experiências de desenvolvimento de tecnologias educacionais sobre crianças e neonatos, com vistas a contribuir para enfermagem pediátrica e neonatal nas dimensões do ensino, da prática e da pesquisa.

\section{OS MATERIAIS EDUCACIONAIS}

0 Grupo de Pesquisa em Enfermagem no Cuidado à Criança e ao Adolescente (GPECCA) da Escola de Enfermagem de Ribeirão Preto da Universidade de São Paulo (EERP/USP) cadastrado no Diretório de Grupos de Pesquisa do CNPq congrega docentes pesquisadores, enfermeiras assistenciais, bolsistas, estudantes de pós-graduação e graduação e tem como uma de suas temáticas de interesse a produção de conhecimentos e tecnologias dirigidas à educação em saúde de crianças e famílias, educação permanente e formação de enfermeiros para o cuidado ao neonato, criança e adolescente e suas famílias.

Como membros do GPECCA, também temos desenvolvido e avaliado o uso de materiais educacionais como jogos, cartilhas e softwares. Durante essa trajetória no desenvolvimento, avaliação e utilização de materiais de ensino, divulgamos nossas experiências em eventos científicos internacionais e nacionais, além de publicações em periódicos indexados, jornais, televisão, rádio e sites.

Pensando nos profissionais de saúde, motivamo-nos à criação e ao uso de novas estratégias e instrumentos de apoio ao processo ensino-aprendizagem dos estudantes de enfermagem e saúde e dos profissionais que atuam na assistência à criança.

Desenvolvemos e utilizamos junto ao Programa de Saúde da Família, um jogo educativo sobre a prevenção de doenças respiratória no seguimento de crianças menores de cinco anos para a capacitação de agentes comunitários de saúde, como produto de uma dissertação de mestrado. A experiência, além de lúdica, proporcionou a participação e interação entre os jogadores, favoreceu o desenvolvimento do processo ensino-aprendizagem, enfocou vivências prévias e se mostrou eficaz para as atividades de educação permanente. 0 trabalho foi publicado em forma de artigo científico, teve dois resumos expandidos em eventos internacionais e um texto em jornal de circulação regional.

Para instrumentalizar as atividades de educação permanente da equipe de enfermagem atuante em unidades 
neonatais, desenvolvemos uma cartilha educativa sobre 0 posicionamento adequado do bebê prematuro em unidade neonatal. Esta apresenta aspectos conceituais sobre o cuidado desenvolvimental além de conter imagens e conteúdos sobre os diferentes tipos de posicionamentos. 0 trabalho foi apresentado em evento científico internacional e atualmente está sendo atualizado para divulgação.

Como resultado de tese de doutorado, desenvolvemos o software educacional Semiotécnica e semiologia do recémnascido pré-termo, o qual foi validado em seu conteúdo e aparência por enfermeiros e especialistas em informática e audiovisual. 0 software apresenta tecnologia avançada, informatizada, interativa e com simulações que permitem a aproximação máxima da realidade, apresentando, gradativamente, a complexidade e as especificidades do recémnascido pré-termo. Por meio de recursos de hipertexto apresenta-se uma série de mídias integradas: fotos, figuras, vídeos e sons; e o conteúdo organizado em necessidades humanas básicas. Está disponibilizado sob a forma de CD-ROM e no momento passa por atualizações e ficará hospedado na internet. Os resultados foram publicados sob a forma de artigo científico em periódico indexado e três resumos expandidos em eventos internacional e nacionais, jornais de universidades pública e privada.

Em continuidade às fases do Processo de Enfermagem, estamos desenvolvendo outro software educacional para auxiliar no processo de ensino do raciocínio clínico para a elaboração do Diagnóstico de Enfermagem em prematuros assistidos em unidade de cuidado intermediário neonatal, o qual será disponibilizado em website. Este é produto de uma tese de doutorado em desenvolvimento, vinculado ao Programa de PósGraduação Enfermagem em Saúde Pública da EERP/USP. Este objeto virtual de aprendizagem inclui revisão teórica da literatura, ensino de três casos clínicos com os respectivos quadros de Risner para construção de cada Diagnóstico de Enfermagem e com quatro casos clínicos para que os alunos possam trabalhar o conteúdo aprendido por meio de simulações de situações reais. 0 software será interativo, pois permite a navegação livre do usuário. Além disso, contará também com recursos audiovisuais (fotos, sons, filmagens) dos sinais e sintomas presentes nos casos clínicos como forma de garantir que o usuário visualize situações reais. Está em fase final de avaliação de seu conteúdo e aparência por enfermeiros e especialistas em informática e audiovisual. Notas prévias deste foram apresentadas em eventos internacionais da área de informática e enfermagem.

Em relação ao processo de educação em saúde, foram elaborados diversos materiais voltados para a clientela, abrangendo o recém-nascido a termo e pré-termo, crianças com fibrose cística e com câncer em fase de tratamento quimioterápico. Os materiais de ensino sobre o bebê a termo e pré-termo tiveram origem nas atividades de educação em saúde com famílias e equipe de enfermagem de alojamento conjunto e unidades neonatais, em que estes participantes ativos no seu processo de ensino e aprendizado elencaram temas que acreditaram ser importantes para o preparo para a alta hospitalar do bebê. Os materiais sobre fibrose cística e quimioterapia são resultados de trabalhos que tiveram os objetivos de analisar as dificuldades que as crianças e os pais enfrentam no ambiente domiciliar, referente a fibrose cística e quimioterapia ambulatorial, sendo apontadas dificuldades de real interesse para assistência à criança e ao adolescente com câncer ou com fibrose cística e sua família.

Entretanto, enfatizamos que, apesar de os materiais a seguir terem como público alvo a criança e sua família, eles foram utilizados na formação dos enfermeiros e em situações de educação permanente da equipe de saúde, com resultados positivos expressos por estudantes e profissionais.

Durante o estágio curricular do Curso de Graduação em Enfermagem, em alojamento conjunto neonatal, criamos material didático que foi utilizado em atividades educativas dirigidas ao preparo das mães para o cuidado do filho recémnascido. Para o desenvolvimento da atividade utilizamos 0 método participativo e técnicas de ensino. Durante o processo de aprendizagem observamos interesse e participação das puérperas. Esta experiência foi divulgada em evento científico nacional, recebendo menção honrosa do Prêmio "Marina de Andrade Rezende" e publicada em revista internacional indexada.

Criamos também o jogo educativo Mamãe e o bebê, sobre os cuidados básicos com o recém-nascido e aleitamento materno, para auxiliar em atividades de educação em saúde junto à clientela. 0 jogo teve excelente aceitação das mães em unidades de alojamento conjunto neonatal, dinamizando o processo ensino-aprendizagem, e contribuiu na aquisição de conhecimentos para o cuidado do filho. Na avaliação realizada pelas mães acerca do jogo, estas expressaram que, se fosse uma "aula normal" elas dormiriam; relataram que durante a atividade com o jogo se sentiram a vontade para perguntar, sem vergonha, e se esqueceram da dor pós-parto e da preocupação causada pelos problemas do cotidiano. Trabalhos sobre o jogo foram divulgados em eventos científicos nacionais e internacional, e o relato de seu desenvolvimento foi publicado em revista internacional indexada, bem como estudo sobre sua utilização. Cópias do jogo foram distribuídas a universidades públicas e a instituições de saúde que prestam atendimento ao parto e ao recém-nascido, por meio de solicitações de profissionais de saúde e docentes.

Especificamente sobre o prematuro, desenvolvemos o jogo educativo Mamãe e o Pequeno Bebê, abordando sobre a alimentação / aleitamento materno, relacionamento familiar, higiene, cuidados diários e cuidados especiais, temáticas essas levantadas pelas mães como necessárias ao preparo para a alta hospitalar de seus filhos em unidades neonatais. A iniciativa da construção deste jogo foi relatada em evento científico nacional, e cópias deste foram entregues em unidades neonatais e cursos 
de graduação em enfermagem mediante solicitação de profissionais.

Ampliando essas experiências, sentimos a necessidade da participação efetiva dos usuários, não só no processo de construção dos conhecimentos, inclusive resgatando o próprio saber das mães, como também no desenvolvimento do material educativo.

Em uma dissertação de mestrado, desenvolvemos material didático-instrucional para auxiliar na orientação da mãe sobre os cuidados ao bebê prematuro, por meio da participação efetiva das mães e da equipe de enfermagem. Os participantes optaram por uma cartilha educativa, no formato perguntaresposta, com ilustrações elucidativas. Os assuntos trabalhados na cartilha são voltados para as práticas cotidianas de cuidados com o prematuro, o aleitamento materno, o relacionamento familiar, situações de risco, serviços de apoio e visitas no domicílio. $^{3}$

A cartilha foi distribuída gratuitamente para pais e familiares de prematuros, estudantes, docentes e profissionais da área de saúde da maioria dos estados brasileiros, bem como foi disponibilizada sua segunda versão no site do Ministério da Saúde (http://bvsms.saude.gov.br/bvs/publicacoes/ Cartilha_cuidados_bebe_premat.pdf) com grande repercussão na comunidade e na mídia, sendo acessada em mais de dez outros sites; foi finalista do prêmio Saúde da Editora Abril, em 2007. Este material demandou uma série de outros trabalhos, divulgados por meio de publicação em periódico internacional, resumos em eventos científicos internacionais e nacionais. Além disso, foram concedidas entrevistas para web, rádio e televisão. Recentemente lançamos a terceira edição dessa cartilha Cuidados com o Bebê Prematuro: orientação para a família prefaciada pelo atual Ministro da Saúde, a qual ao longo dos anos incorporou as sugestões emitidas pelos usuários, além da Declaração Universal dos Direitos do Bebê Prematuro. Esta nova edição da cartilha também está online no site do Ministério da Saúde (http://bvsms.saude.gov.br/bvs/publicacoes/ cuidados_bebe_prematuro_3ed.pdf).

Pensando na orientação de famílias e crianças em condições crônicas, desenvolvemos duas cartilhas educativas. A primeira delas trata da assistência à criança com câncer. 0 conteúdo teórico da cartilha emergiu a partir de um trabalho desenvolvido com os pais de filhos com câncer para analisar as dificuldades que os mesmos enfrentavam no ambiente domiciliar, quando seus filhos eram submetidos à quimioterapia ambulatorial. A partir daí os dados foram reunidos e originaram a cartilha Quimioterapia: Orientações para a Família. A cartilha aborda aspectos conceituais sobre câncer infantil e quimioterapia; trata também das reações adversas do tratamento quimioterápico e dá orientações do que se deve fazer nas seguintes situações: cansaço, náusea e vômito, mucosite, perda de apetite, alteração no peso, alopecia, depressão, alteração do humor, problemas para dormir e imunossupressão. Ainda aborda aspectos relacionados ao uso de máscaras, dificuldades socioeconômicas, a escola e mudanças na estrutura familiar. 0 trabalho também foi publicado em jornais, revista internacional indexada e em eventos internacionais e nacionais.

Outra cartilha educativa trata da fibrose cística: Temos Fibrose Cística - e agora? Foi elaborada para crianças e adolescentes portadores de fibrose cística e seus familiares. Aborda aspectos conceituais sobre a doença, o diagnóstico, os sinais e sintomas, os tratamentos, a alimentação e aspectos relacionados ao meio social da criança. 0 trabalho foi apresentando em evento nacional.

Para educação em saúde da clientela, estamos desenvolvendo como produto de uma tese de doutorado vinculada ao Programa de Pós-Graduação Enfermagem em Saúde Pública da EERP/USP, um website sobre a amamentação, com o objetivo de auxiliar mães de prematuros e suas famílias no processo do aleitamento materno. Dentre os conteúdos que o website abordará estão: as características e importância do leite materno, posicionamento correto do bebê durante e após a amamentação, frequência das mamadas, relactação e translatação, relacionamento entre mãe-filho e família durante este período, ordenha manual de leite e armazenamento, oferecimento de leite por copinho, além de possíveis dificuldades encontradas por mães e familiares do pré-termo durante este processo. Este tema será abordado de forma interativa com os usuários, por meio de navegação livre destes. Utilizaremos também recursos audiovisuais, tais como animações, filmagens, sons e fotos. Haverá um espaço para discussão online, com abertura para a manifestação de dúvidas e experiências anteriores, entre os participantes e pesquisadora. Os jogos educativos, integrados ao website, também serão utilizados com a finalidade de promover a interação entre usuários além de permitir o aprendizado dos conteúdos.

Estas iniciativas demonstram que o enfermeiro, por meio de seu papel de educador, contribui para 0 empoderamento da clientela com vistas à melhora da qualidade de vida. Trabalhar com as questões educacionais junto à população pode significar amadurecimento profissional e pessoal, que, além de ampliar seu conhecimento, pode auxiliar a expansão de aprendizado e reflexões acerca de questões éticas que envolvem esta atividade. ${ }^{4} \mathrm{C}$ abe ressaltar que alguns aspectos podem ser limitantes, situaç̃̃es em que 0 acesso ao computador e dificuldades no seu manejo podem estar presentes, embora os softwares desenvolvidos sejam de fácil navegação.

0 Ministério da Saúde recomenda que a produção de um material didático precisa ser cuidadosa em relação à linguagem de seus textos, voltada ao público-alvo. É importante que a linguagem seja clara, objetiva e coloquial, adequada às características da clientela. Isto permite uma leitura leve e agradável, de fácil compreensão. ${ }^{5}$ 
Quando o material educativo é de fácil compreensão, 0 cliente adquire maior conhecimento, o material é capaz de suscitar mudanças de atitudes e desenvolvimento de habilidades, além de favorecer a autonomia, tomada de decisão e 0 entendimento de que as suas ações influenciam no padrão de saúde. $^{6}$

Desde 1992, o Ministério da Saúde tem recomendado que a população se envolva com os programas de saúde, com o objetivo de promover transformações conceituais na compreensão da saúde, visando à qualidade e compromisso com a vida, e não, simplesmente, à ausência de enfermidades, gerando atitudes e procedimentos novos diante dos problemas relacionados com a doença, de modo que a saúde seja encarada como responsabilidade de todos e não somente atribuição governamental. ${ }^{7}$

Neste sentido, a prática da educação em saúde deve estar comprometida com a transformação social do indivíduo envolvido no processo educativo, de forma coerente, contínua e sensibilizada com o desenvolvimento social e político da população. ${ }^{8}$

Tanto a construção da maioria dos materiais educacionais como as atividades educacionais auxiliadas por destes têm sido fundamentadas em referencial pedagógico, tendo por base a problematização de Paulo Freire, dentre outras metodologias ativas de aprendizagem, cujos objetivos são a transformação social, a troca de experiências, o questionamento, a individualização e a humanização; comprometem-se com uma programação, com conteúdos que advém das colocações de sua existência, desafiando o educando a buscar respostas. Desse modo, buscam juntos, educador e educandos, mediatizados pelo ambiente e pela realidade, o conteúdo a ser estudado. 0 uso destas abordagens tem favorecido a participação ativa dos sujeitos no processo, desde o questionamento da sua realidade, a busca de respostas, a escolha do tipo de material (formato, tipo de letra, imagens, cores, linguagem) a ser construído, passando pelo levantamento dos temas a serem trabalhados até a avaliação destes materiais criados. 0 embasamento da pedagogia problematizadora tem se mostrado adequado no desenvolvimento de materiais com tecnologia da informática por possibilitar a aquisição do conhecimento de forma não linear e implicar a participação dos agentes na construção de seus conhecimentos e a troca de experiências; as simulações buscam retratar situações cotidianas oferecendo reflexão e ação e feedback do aprendizado. ${ }^{9}$

Algumas escolas de enfermagem ainda hoje utilizam pedagogias tradicionais em que o estudante não é ativo no processo de construção dos seus próprios conhecimentos. 0 processo de formação do enfermeiro deve possibilitar que 0 indivíduo seja crítico-reflexivo, preparando-o para ser agente transformador da sociedade em que vive, comprometido com os problemas da população e com a melhoria da qualidade de saúde. Assim, o momento é de repensar a prática de formação de enfermeiros envolvendo a inserção de novos modelos pedagógicos que possibilite o desenvolvimento do estudante para se tornar um enfermeiro crítico-reflexivo e político para problemas do contexto atual. ${ }^{10}$

0 material educativo deve permitir partir do contexto do aprendiz, de suas experiências e vivências para que, a partir delas, ele possa construir o seu próprio conhecimento. ${ }^{5}$

Durante esta trajetória de desenvolvimento e avaliação de materiais educativos, tivemos oportunidade de utilizar estes materiais com os estudantes de graduação, profissionais de enfermagem que demonstraram interesse e motivação, tanto no uso para seu próprio aprendizado quanto na utilização educativa junto aos usuários.

Alguns educadores têm procurado agregar novos conhecimentos em práticas de ensino e os recursos adicionais às práticas educativas. Ressaltamos a importância de 0 estudante ter contato com materiais educacionais durante sua formação.

No Brasil, apesar da escassez de material educativo computacional que pode auxiliar na ligação rápida da teoria à prática, o número de iniciativas de desenvolvimento tem se ampliado especialmente neste século.

As metodologias ativas de aprendizagem se propõem a substituir a memorização e a simples transferência de informações e de habilidades, pela construção do conhecimento a partir da vivência de situações reais ou simuladas da prática profissional, estimulando as capacidades de análise crítica e reflexiva e o aprender.

A utilização da tecnologia no processo de ensinoaprendizagem está plenamente justificada se levarmos em conta que um dos objetivos básicos da educação é preparar os estudantes para serem cidadãos de uma sociedade plural, democrática e tecnologicamente avançada. ${ }^{11}$

A formação do enfermeiro não se encerra no final da graduação, e este deve sempre aprender e pesquisar sobre a atualização tecnológica conceitual para ser competente na integração e aplicação dos mesmos. ${ }^{12}$

Os recursos tecnológicos são ferramentas necessárias ao enfermeiro, pois contribuirá para um gerenciamento da assistência de enfermagem de forma humanizada, no âmbito da qualidade, eficácia, efetividade e segurança, de maneira que possa garantir os resultados do uso adequado da tecnologia para os quais ela foi desenvolvida e incorporada. A equipe de enfermagem terá assim maior segurança, redução de estresse e, especialmente, o desempenho e performance operacional necessários à otimização desses recursos nos espaços, atividades e intervenções onde estão instalados. ${ }^{13}$

A área da saúde precisa e deve utilizar-se dos recursos tecnológicos; porém, os profissionais não devem esquecer que o computador jamais substituirá a essência humana. Os avanç̧os tecnológicos devem favorecer o resgate da natureza humana. Torna-se necessário que os enfermeiros façam uma reflexão sobre suas posturas enquanto seres humanos que prestam cuidados. A produção tecnológica da Enfermagem descreve 
uma ampla variedade de materiais que se caracterizam pelo custo reduzido, pela capacidade de satisfazer necessidades básicas mais carentes, pelo uso racional e por um elevado grau de adaptação ao ambiente local, cultural e social. ${ }^{2}$

0 objetivo dos materiais educativos deve ser o de facilitar o trabalho da equipe de saúde na comunicação e orientação de pacientes e familiares. Estes materiais subsidiam a orientação verbal dos profissionais de saúde aos familiares e pacientes e uniformizam as orientações a serem realizadas sobre os cuidados. ${ }^{14}$

Sendo assim, é importante que não seja somente entregue o material impresso, mas que a comunicação entre a enfermagem e a família seja eficiente, com trocas de experiências entre familiares, nos quais os participantes sejam ativos no processo de construção de seus conhecimentos. Portanto, esta iniciativa deve estar inserida em um contexto maior de educação em saúde, possibilitando um continuum da assistência em saúde.

Na perspectiva do cliente, acreditamos que os materiais educacionais proporcionam que ele tenha participação crítica ativa tanto na construção de alguns materiais como na sua execução. Isto corrobora o nosso pensamento de que o cliente é um ser social, sujeito de suas ações, já que tem anseios e esperanças, seu próprio modo de aceitar as inovações e estabelecer relação com seus semelhantes. Assim, o pesquisador deve sempre se remeter à construção de tecnologias que integram o ser, o pensar, o fazer, mobilizando ações de cuidado humano. ${ }^{15}$

\section{CONSIDERAÇÕES FINAIS}

A universidade pública tem um compromisso com a sociedade: contribuir para sua transformação e formar cidadãos ativos e críticos de sua realidade. Além da produção, construção e divulgação do conhecimento científico, é fundamental que ela forme trabalhadores qualificados, eleve o nível cultural da sociedade e participe da resolução dos problemas sociais.

Assim, a preocupação inerente em nosso grupo de pesquisa é que, desde o desenvolvimento dos materiais educacionais até sua utilização, estes sejam realizados de forma participativa, tornando os sujeitos ativos no processo ensinoaprendizagem, sejam alunos de enfermagem, profissionais de saúde ou clientela, em uma relação horizontal que estimule e possibilite a troca de experiência, o pensamento crítico-reflexivo e a construção conjunta de conhecimento, sendo estes sujeitos agentes multiplicadores de conhecimento e transformadores de sua realidade.

Consideramos que os materiais educacionais criados pelo GPECCA favorecem que estudantes, profissionais de saúde e clientes vivenciem o processo ensino-aprendizagem de forma estimulante, facilitando o esclarecimento de dúvidas e transformando a aprendizagem com situações atrativas, inovadoras e ilustrativas. Precisamos ir além do desenvolvimento de materiais educacionais, pois temos a responsabilidade e 0 compromisso, enquanto membros do grupo de pesquisa, de, a partir das experiências existentes tanto no desenvolvimento quanto na utilização de tecnologias no ensino de Enfermagem, avaliar não apenas os produtos tecnológicos construídos, mas também no que estes têm contribuído para o processo ensinoaprendizagem, tendo como foco o seu impacto.

Mais do que disponibilizar uma coletânea de materiais educacionais, apresentamos referenciais que auxiliam 0 desenvolvimento das potencialidades dos estudantes, equipe de enfermagem, criança e família. Nas atividades educativas de todos os sujeitos envolvidos em cada um dos objetos criados, esperamos que sejam catalisadores e estimulem 0 interesse para explorar os materiais educativos.

\section{NOTA}

Trabalho vinculado ao Grupo de Pesquisa em Enfermagem no Cuidado à Criança e ao Adolescente (GPECCA) do Departamento de Enfermagem Materno-Infantil e Saúde Pública da Escola de Enfermagem de Ribeirão Preto da Universidade de São Paulo (EERP-USP), Centro Colaborador da OMS para o desenvolvimento da pesquisa em enfermagem.

\section{Autores de Rodapé}

Fernanda dos Santos Nogueira de Góes

Enfermeira. Doutoranda do Programa de Pós-Graduação Enfermagem em Saúde Pública da Escola de Enfermagem de Ribeirão Preto da Universidade de São Paulo. Ribeirão Preto-SP.Brasil. E-mail: fersnogueira@usp.br;

\section{Geovana Magahães Ferecini}

Enfermeira. Doutoranda do Programa de Pós-Graduação Enfermagem em Saúde Pública da Escola de Enfermagem de Ribeirão Preto da Universidade de São Paulo. Ribeirão Preto - SP. Brasil. E-mail: geovana_ferecini@yahoo.com.br

\section{Lucila Castanheira Nascimento}

Enfermeira. Professora Doutora do Departamento de Enfermagem MaternoInfantil e Saúde Pública da Escola de Enfermagem de Ribeirão Preto da Universidade deSãoPaulo. RibeirãoPreto-SP.Brasil.E-mail: lucila@eerp.usp.br

Maria Cândida Carvalho Furtado

Enfermeira. Professora Doutora do Departamento de Enfermagem MaternoInfantil e Saúde Pública da Escola de Enfermagem de Ribeirão Preto da Universidade de São Paulo. Ribeirão Preto - SP. Brasil. E-mail: mcandida@eerp.usp.br

\section{REFERÊNCIAS}

1.Padalino Y, Peres HHC. E-Learning: estudocomparativo da apreensão do conhecimento entre enfermeiros. Rev Latino-am Enfermagem. 2007 jun; 15(3): 397-403.

2.Barra DCC, Nascimento ERP, Martins JJ, Albuquerque GL, Erdmann AL. Evolução histórica e impacto da tecnologia na área da saúde e da enfermagem. Rev Eletr Enf [periódico na internet]. 2006; [citado 2006]; 8(3): 422-30. Disponível em: http://www.fen.ufg.br/revista/revista8_3/ v8n3a13.htm

3.Fonseca LMM, Scochi CGS, Rocha SMM, Leite AM. Cartilha educativa para orientação materna sobre os cuidados com o bebê prematuro. Rev Latino-am Enfermagem. 2004 fev; 12(1): 65-75. 
4.Gomes CK, Freitag PLM. Tecnologia assistiva em saúde para cegos: enfoque na prevenção de drogas. Esc Anna Nery. 2007 dez; 11(4): 67781.

5.Ministério da Saúde (BR). Elaboração de material didático impresso para programas de formação a distância: orientações aos autores. Brasília(DF):EAD/ENSP / FIOCRUZ; 2005.

6.Serxner S. How readability of material affects outcomes. J Vasc Nurs. 2000 Sep; 18(3): 97-101.

7.Ministério da Saúde (BR). Educação para a saúde: plano estratégico. Brasília (DF); 1992.

8. Torres GV, Enders BC. Atividades educativas na prevenção da AIDS em uma rede básica municipal de saúde: participação do enfermeiro. Rev Latino-am Enfermagem. 1999 abr; 7(2): 71-77.

9.Fonseca LMM, Leite AM, Mello DF, Dalri MCB, Scochi CGS. Semiotécnica e semiologia do recém-nascido pré-termo: avaliação de um software educacional. Acta Paul Enferm. 2008 jun; 21 (4): 543-8.

10.Rodrigues J, Zagonel IPS, Mantovani MF. Alternativas para a prática docente no ensino superior de enfermagem. Esc Anna Nery. 2007 jun; 11(2): 313-17.

11.Maiztegui A, et al. Papel de la tecnología en la educación científica: una dimensión olvidada. Rev Iberoam Educ. 2002 jan; 28: 129-55.

12.Nietsche EA. Tecnologia emancipatória: possibilidade ou impossibilidade para a práxis de enfermagem? ljuí(RS): Unijuí; 2000.

13.Arone EM, Cunha ICKO. Avaliação tecnológica como competência do enfermeiro: reflexões e pressupostos no cenário da ciência e tecnologia. Rev Bras Enferm. 2006 jul; 59(4): 569-72.

14.Echer IC. Elaboração de manuais de orientação para o cuidado em saúde. Rev Latino-am Enfermagem. 2005 set; 13(5): 754-57.

15.Erdmann AL, Andrade SR, Mello ALSF, Meirelles BHS. Gestão das práticas de saúde na perspectiva do cuidado complexo. Texto\&Contexto Enferm. 2006 jul; 15(3): 178-82. 